\title{
Minimum linewidth of surface plasmon resonance in doped ZnO nanocrystals
}

\author{
Christophe Delerue* \\ Univ. Lille, CNRS, Centrale Lille, ISEN, Univ. Valenciennes, UMR 8520-IEMN,F-59000 \\ Lille, France \\ E-mail: christophe.delerue@iemn.univ-lille1.fr
}

KEYWORDS: plasmon, nanocrystal, nanoscale physics, zinc oxide

\begin{abstract}
The optical response of $\mathrm{ZnO}$ nanocrystals (NCs) doped with $\mathrm{Al}(\mathrm{Ga})$ impurities is calculated using a model that incorporates the effects of quantum confinement, dielectric mismatch, surface and ionized impurity scattering. For dopant concentrations of a few percent, the NC polarizability is dominated by a localized surface plasmon resonance (LSPR) in the infrared (IR) which follows the Drude-Lorentz law for NC diameter above $\sim 10 \mathrm{~nm}$ but is strongly blue-shifted for smaller diameters due to quantum confinement effects. The intrinsic width of the LSPR peak is calculated in order to characterize plasmon losses induced by ionized impurity scattering. Widths below $80 \mathrm{meV}$ are found in the best cases, in agreement with the lowest values recently measured on single NCs. These results confirm that doped $\mathrm{ZnO}$ NCs are very promising for the development of IR plasmonics. The width of the LSPR peak strongly increases when dopants are placed near the surface of the NCs, or when additional fixed charges are present.
\end{abstract}

\footnotetext{
*To whom correspondence should be addressed
} 
Metallic plasmonic nanocrystals (NCs) have been intensively studied for light-harvesting ${ }^{1}$ and bio-sensing ${ }^{2}$ applications. At the local surface plasmon resonance (LSPR), the enhanced electric fields in the vicinity of the NC surface are used to concentrate far-field radiations. For noble metals such as gold and silver, the LSPR lies in the visible range due to the high (fixed) concentration of free carriers. Alternatively, NCs of heavily-doped (degenerate) semiconductors recently emerged as promising materials for the extension of plasmonics to the infrared (IR) range. ${ }^{3-7}$ This was demonstrated with NCs of copper chalcogenide semiconductors, ${ }^{3,8-11} \mathrm{P}$ - or B-doped $\mathrm{Si},{ }^{12-14}$ and oxide semiconductors such as $\mathrm{SnO}_{2}: \mathrm{Sb}^{15}{ }^{15} \mathrm{In}_{2} \mathrm{O}_{3}: \mathrm{Sn},{ }^{16-19}$ $\mathrm{In}_{2} \mathrm{O}_{3}: \mathrm{Ce},{ }^{20} \mathrm{ZnO}: \mathrm{Al}(\mathrm{AZO}),{ }^{21-23} \mathrm{ZnO}: \mathrm{Ga}(\mathrm{GZO}){ }^{24,25}$ and $\mathrm{ZnO}: \mathrm{In} .{ }^{26}$ In these NCs, the LSPR energy can be tuned by changing the free carrier density through the control of the dopant concentration. ${ }^{3,21,27-30}$ This opens the door to technological applications in chemical sensing, bio-detection, thermography, IR optical telecommunication and imaging. In particular, plasmonic responses resonant with molecular vibrational modes can be employed to enhance the sensitivity of chemical sensing through IR spectroscopy. ${ }^{31}$

A main challenge in plasmonics is to reduce losses in order to increase the plasmon lifetime and to enhance the local electric field. ${ }^{32}$ The damping of the free electron oscillation can be probed through the measurement of the LSPR peak linewidth on single NCs. ${ }^{33}$ Interestingly, recent investigations on single AZO NCs report linewidths as narrow as $600 \mathrm{~cm}^{-1}(74 \mathrm{meV})$ which is markedly less than for gold nanoparticles. ${ }^{23}$ This raises the fundamental question of the prediction of the intrinsic linewidth of the LSPR peak in doped oxide semiconductor NCs. In the range of dopant concentration used in these NCs, typically of the order of a few percent, damping is due to ionized impurity scattering $5,17,20,23,34,35$ even if the role of surface scattering is mentioned. ${ }^{36}$ In previous works on the LSPR in doped semiconductor $\mathrm{NCs},{ }^{3,7,17,20,37-40}$ the effect of impurity scattering on the linewidth was either introduced as a free parameter or described using a Drude-Lorentz theory based on bulk properties. While it was shown experimentally ${ }^{40}$ and theoretically ${ }^{39,40}$ that the LSPR in semiconductor NCs can be strongly shifted in energy by the quantum confinement, the effects of spatial, quantum 
and dielectric confinements on impurity scattering processes are presently unknown. Indeed, in NCs, the quantization of the electronic levels could perturb the scattering channels, and electron-electron and electron-ion interactions could be influenced by the presence of the surface. ${ }^{41}$ In addition, the position of the impurities in the NCs is expected to play an important role, not only on the energy ${ }^{7,39}$ but also on the linewidth of the LSPR peak. As a consequence, the minimum value of the Full Width at Half Maximum (FWHM) of the LSPR peak in doped semiconductor NCs is still unknown from a theoretical point of view.

In this paper, I present calculations of the dynamic polarizability of $\mathrm{Ga}(\mathrm{Al})$-doped $\mathrm{ZnO}$ NCs in order to predict the intrinsic linewidth of the LSPR peaks. The theoretical methodology allows to work on NCs with diameter up to $14 \mathrm{~nm}$ and with a number of free carriers up to 700 but nevertheless describes the dopant impurity potential at the microscopic level and includes quantum and dielectric confinement effects on both the electronic structure and the impurity scattering. The calculations confirm the important role of the quantum confinement on the LSPR energy. ${ }^{39,40,42}$ On the contrary, it is found that the calculated LSPR linewidth has a little dependence on the NC size. The narrowest linewidths just limited by ionized impurity scattering are predicted in good agreement with the smallest experimental values measured on single $\mathrm{ZnO} \mathrm{NCs}^{23}$ confirming the great potential of oxide $\mathrm{NCs}$ for plasmonic applications. It is also shown that the FWHM is considerably increased when additional fixed charges are introduced in the NCs, or when the dopants are preferentially placed near the surface of the NCs. These results provide lower limits for the linewidths and useful indications toward their minimization in doped oxide NC systems, and in doped semiconductor NCs in general.

The calculations are based on a single-band theory in the Random-Phase Approximation (RPA). $\mathrm{ZnO}$ is described by a conduction band of effective mass $m_{e}=0.25 m_{0}$ where $m_{0}$ is the free-electron mass. The small effective-mass anisotropies induced by the anisotropic wurtzite lattice of $\mathrm{ZnO}$ can be safely neglected (Supporting Information). The effective-mass theory provides a very good description of donor impurities in bulk $\mathrm{ZnO}$, in particular for the 
electron binding energy. ${ }^{43}$ The chemical shifts on $\mathrm{Al}$ and Ga impurities are small $(\lesssim 3 \mathrm{meV})$ and their influence was found to be negligible on the LSPR spectra. The quantum states $\Psi_{n}$ of energy $E_{n}$ are calculated for spherical NCs (radius $R$ ) assuming an infinite potential well at the surface. For that purpose, the Schrödinger and Poisson equations are solved selfconsistently on a regular three-dimensional grid. In order to work with linear equations, the problem is rewritten in a Tight-Binding (TB) formalism characterized by a single $s$ orbital $\phi_{i}$ per site $i$ and a nearest-neighbor hopping $t$. For convenience, a grid was built on a zinc-blende lattice but it was checked that the same results can be obtained using a cubic lattice. The hopping term is chosen to give the correct effective mass in the bulk, i.e., $t=-4 \hbar^{2} /\left(m_{e} a^{2}\right)$ where $a$ is the lattice parameter. Dopants ( $\mathrm{Ga}$ or $\mathrm{Al}$ ) are placed at random positions on the grid, excluding the sites at the surface. The Coulomb potential induced by a fixed charge $+e$ at each impurity nucleus is added to the bare TB Hamiltonian, ${ }^{37,41}$ as well as the potential induced by the free electrons (hereafter, those brought by the dopants). The total potential is solved self-consistently. ${ }^{44}$ The dielectric response inside the NC is described by the static dielectric constant of bulk $\mathrm{ZnO}$, which is totally justified for $\mathrm{NC}$ diameters above $4 \mathrm{~nm} .{ }^{41}$

We calculate the polarizability of a $\mathrm{NC}$ along a direction $\mathbf{u}$ at the photon energy $h \nu$ as $\alpha(h \nu)=-e^{2} \sum_{i, j} \chi_{i j}^{\mathrm{rpa}}\left(\mathbf{R}_{i} \cdot \mathbf{u}\right)\left(\mathbf{R}_{j} \cdot \mathbf{u}\right)$, where the sum is over all sites $i$ and $j$ of position $\mathbf{R}_{i}$ and $\mathbf{R}_{j}$, and $\chi^{\mathrm{rpa}}$ is the RPA susceptibility matrix. ${ }^{45} \chi^{\mathrm{rpa}}$ is given by $\chi^{0} \varepsilon^{-1}$, where $\varepsilon=I-V \chi^{0}$ is the dielectric matrix ${ }^{46} I$ is the identity matrix, $V$ is the matrix of the bare electron-electron interaction $\left(V_{i j}=e^{2} /\left|\mathbf{R}_{i}-\mathbf{R}_{j}\right|\right){ }^{47,48}$ and $\chi^{0}$ is the independent-particle susceptibility matrix ${ }^{46}$

$$
\chi_{i j}^{0}=\chi_{i j}^{v}+\sum_{n, n^{\prime}} \frac{\left(f_{n}-f_{n^{\prime}}\right) c_{n, i} c_{n^{\prime}, i}^{*} c_{n, j} c_{n^{\prime}, j}^{*}}{h \nu-\left(E_{n^{\prime}}-E_{n}\right)+i \eta} .
$$

The first term $\chi^{v}$ represents the response of the valence electrons and the second one of the free electrons. $f_{n}$ is the electronic population at $T=300 \mathrm{~K}$ on the state $\Psi_{n}=\sum_{i} c_{n, i} \phi_{i}$. The matrix $\chi^{v}$ is restricted to on-site and nearest-neighbor terms. The latter are all given by a single term equal to $\left(\varepsilon_{b}^{\infty}-1\right) a /(4 \pi)$ where $\varepsilon_{b}^{\infty}$ is the dynamical dielectric constant of bulk 
$\mathrm{ZnO}$ at the frequency $\nu .{ }^{49}$ The on-site terms are determined by the relations $\sum_{j} \chi_{i j}^{v}=0$ that come from the charge conservation in the system. ${ }^{48}$ This method provides a good description of the dielectric response to slowly-variable potentials in semiconductor NCs. ${ }^{41,45,48}$ Details on the methodology can be found in these references. The computation of $\chi^{\mathrm{rpa}}$ typically scales as $n_{e} V^{3}$ where $n_{e}$ is the number of free electrons and $V$ is the $\mathrm{NC}$ volume. In spite of this unfavorable scaling, it has been possible to investigate NCs with diameter up to $14 \mathrm{~nm}$. Finally, it is worth noting that surface scattering processes are naturally described in these calculations.

In the following, all presented spectra have been obtained with a broadening $\eta$ of 0.01 $\mathrm{eV}$ (the role of this value will be discussed later). For the sake of comparison, the spectra correspond to $\alpha(h \nu) / R^{3}$ as the polarizability $\alpha(h \nu)$ scales as $R^{3}$. The dopant concentration is defined as the ratio between the number of dopants and the number of cations in the undoped NC.

Typical spectra of the dynamical polarizability are shown in Figs. 1a,b for NCs characterized by a uniform doping, and a ratio between the numbers of free carriers and dopants (doping efficiency) of $100 \%$. For a number of dopants typically larger than $10,{ }^{37}$ each absorption spectrum $[\propto$ imaginary part of $\alpha(h \nu)]$ is characterized by a broad peak in an energy region where the real part of $\alpha(h \nu)$ becomes negative. The integral of the absorption cross section, $\sim \int_{0}^{\infty} \varepsilon \operatorname{Im}[\alpha(\varepsilon)] d \varepsilon$, is proportional to the number of free carriers (Supporting Information Fig. S1) ${ }^{39}$ At a dopant concentration of $0.6 \%$, the LSPR peak for the NC diameter $D$ of $6 \mathrm{~nm}$ exhibits (Fig. 1a) some fine structures (small oscillations) which are less visible for $D=12 \mathrm{~nm}$ (Fig. 1b). This is explained by a statistical effect, since the plasmon in the larger NC comes from the coupling between oscillators induced by 240 free electrons, compared to 30 in the smaller NC. As a result, the LSPR peak for $D=6 \mathrm{~nm}$ still contains some small structures that come from single-particle transitions. ${ }^{37}$ Disorder effects (e.g., related to dopant positions) are also less averaged in a NC containing 30 impurities than 240.

The evolution of the LSPR peak energy (taken at the peak maximum) is shown in Fig. 1c. 

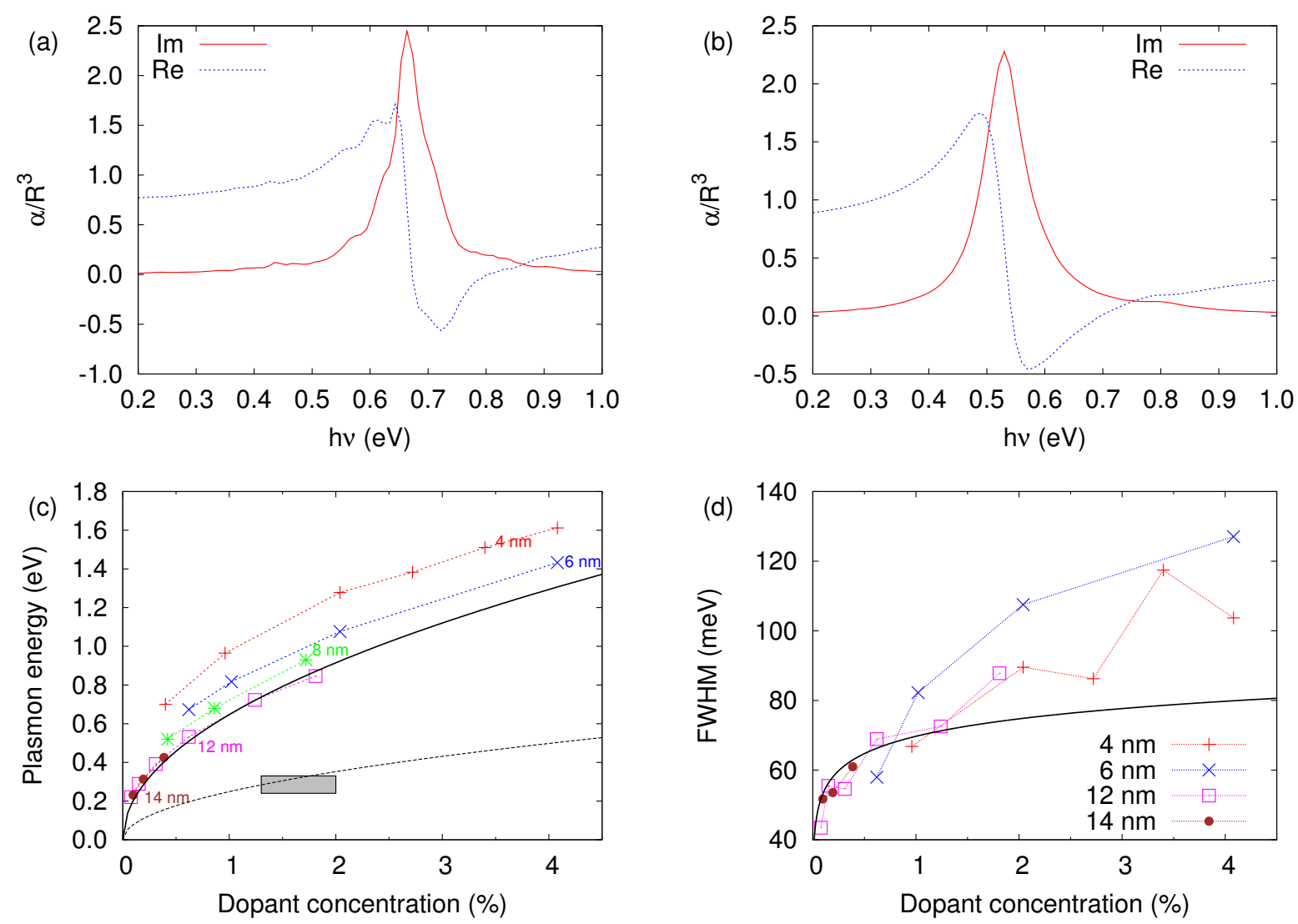

Figure 1: Real (Re) and Imaginary ( $\operatorname{Im})$ parts of $\alpha(h \nu) / R^{3}$ for NCs doped at a concentration of $0.6 \%: D=2 R=6 \mathrm{~nm}$ and 30 dopants (a), $D=12 \mathrm{~nm}$ and 240 dopants (b). (c) Energy of the LSPR peak vs doping concentration for $D=4 \mathrm{~nm}($ red + ), $6 \mathrm{~nm}$ (blue $\times$ ), $8 \mathrm{~nm}$ (green *), $12 \mathrm{~nm}$ (magenta squares), and $14 \mathrm{~nm}$ (brown circles). The black solid line represents the LSPR energy predicted by the Drude-Lorentz law for a surrounding medium of dielectric constant $\varepsilon_{m}=1$. The black dashed line shows the same curve but for a doping efficiency of $20 \%$ and $\varepsilon_{m}=2.0$. The gray rectangle indicates the region containing the LSPR peak energies measured on single AZO NCs in Ref. ${ }^{23}$ (d) Contribution of ionized impurity scattering to the FWHM of the LSPR peak for $D=4 \mathrm{~nm}($ red + ), $6 \mathrm{~nm}$ (blue $\times), 12 \mathrm{~nm}$ (magenta squares), and $14 \mathrm{~nm}$ (brown circles). The black dotted line represents the same contribution deduced from the Brooks-Herring theory for bulk ZnO. The phonon scattering contribution to the FWHM which must be added is estimated of the order of $10 \mathrm{meV}$.

For $D=12 \mathrm{~nm}$ and $14 \mathrm{~nm}$, the energy is very well described by the Drude-Lorentz theory which gives

$$
\omega_{\mathrm{DL}} \approx \sqrt{\frac{4 \pi n e^{2}}{m_{e}\left(\varepsilon_{b}^{\infty}+2 \varepsilon_{m}\right)}},
$$

where $n$ is the volume density of free carriers and $\varepsilon_{m}$ is the dynamic relative permittivity of 
the surrounding medium (here equal to unity, except otherwise stated). Equation 2 is valid at the condition $\gamma^{2} \ll \omega_{\mathrm{DL}}^{2}$ where $\gamma$ is the damping factor. ${ }^{3}$ In this situation, $\gamma$ coincides with the FWHM of the LSPR peak.

The calculated LSPR peaks are clearly shifted to higher energy with respect to $\omega_{\mathrm{DL}}$ in the smallest NCs, as already shown in previous works on doped semiconductor NCs. ${ }^{37,39,40}$ In the present case, the blue shift can be interpreted by the effect of the quantum confinement on the plasmon. ${ }^{39,40}$ As a matter of fact, we have checked that, if we tune the effective mass of the conduction band, the difference between the calculated values and $\omega_{\mathrm{DL}}$ scales as $1 / m_{e}$ (Supporting Information Fig. S2). Confinement effects are found negligible for diameters above $12 \mathrm{~nm}$, in excellent agreement with the results of Refs. ${ }^{40,42}$ In accordance with these findings, recent measurements on single AZO NCs demonstrate the absence of correlation between the LSPR peak energy and the NC size for $D>10 \mathrm{~nm} \cdot{ }^{23}$

In the single-NC experiments of Ref., ${ }^{23}$ the $\mathrm{Al}$ concentration was evaluated between $1.3 \%$ and $2.0 \%$, with an average of $1.6 \%$, and the LSPR peak was found between $0.24 \mathrm{eV}$ and $0.33 \mathrm{eV}$ (region depicted by a gray rectangle in Fig. 1c). These values are consistent with ensemble measurements made on AZO and GZO NCs. ${ }^{21,22,24,25}$ In order to interpret LSPR peak energies in this range, it is necessary to consider two effects: 1) the average dielectric constant $\varepsilon_{m}$ of the environment is larger than unity; 2) the doping efficiency can be well below $100 \%$, as discussed in previous studies. ${ }^{21,25,42,50-52}$ For example, the Drude-Lorentz law for $\varepsilon_{m}=2.0$ and a doping efficiency of $20 \%$ gives LSPR peak energies compatible with the experimental observations (Fig. 1c). It is important to realize that, in real experimental situations, the dielectric environment of the NCs is not homogeneous and is likely to change from $\mathrm{NC}$ to NC.

The FWHM of the LSPR peak was obtained by fitting $\operatorname{Im}[\alpha(h \nu)]$ with a Lorentzian function and by extracting its width $F(\eta)$. Using the fact that $F(\eta)$ is a linear function of the broadening parameter $\eta$ (Fig. S3, Supporting Information), it is easy to determine the limit $F(\eta \rightarrow 0)$ that gives us the intrinsic FWHM presented in Fig. 1d. Remarkably, if we 
perform the same calculations but in a jellium model, i.e., after replacement of the dopant ions by an equivalent homogeneous positive charge, $F(0)$ is very small whereas, in the model with real dopants, values of a few tens or hundreds of meV are obtained (Fig. S3, Supporting Information). This confirms the essential role of ionized impurity scattering on the plasmon damping. ${ }^{5,17,20,23,34,35}$ In fact, the scattering of the electronic waves by the dopants leads to a profound reorganization of the single-particle intraband transitions (Supporting Information, Fig. S4) in such a way that some of them become resonant with the LSPR and provide a direct channel for the plasmon decay (Landau damping).

Figures 1c,d show that the FWHM of the LSPR peak is always small compared to the LSPR energy (implying $\gamma^{2} \ll \omega_{\mathrm{DL}}^{2}$ ), explaining why the LSPR energies agree with Eq. 2 when confinement effects become negligible. In addition, the results show that surface scattering is negligible for the dopant concentrations investigated here $\left(>10^{20} \mathrm{~cm}^{-3}\right)$, at least for NCs with a spherical shape in absence of surface roughness.

The linewidths presented in Fig. 1d do not include the contribution $\left(\gamma_{p h}\right)$ from phonon scattering (at $300 \mathrm{~K}$, mainly polar phonon scattering ${ }^{53}$ ). This contribution can be estimated from the carrier mobility $\mu$ measured in best-quality undoped (lightly doped) $\mathrm{ZnO}$ samples. A mobility of $440 \mathrm{~cm}^{2} /(\mathrm{Vs})$ was found in Ref. ${ }^{53}$ from Hall measurements confirmed by the theory, in good agreement with terahertz time domain spectroscopy measurements of Ref. ${ }^{54}\left[493 \mathrm{~cm}^{2} /(\mathrm{Vs})\right]$. This gives a damping factor $\gamma_{p h}=\hbar e /\left(m_{e} \mu\right)$ of about $10 \mathrm{meV}$. This phonon contribution is small compared to the FWHMs of Fig. 1d but becomes non negligible for the lowest dopant concentrations $(<0.5 \%)$, and will become dominant for concentrations below $0.1 \%$. In addition, if the number of dopants in a $\mathrm{NC}$ is of the order of 10 or below, a regime is reached where the LSPR peak strongly overlaps with intraband transition peaks, giving rise to more complex lineshapes ${ }^{37}$ (see also Refs. ${ }^{22,55}$ for single-particle spectra). This regime was not considered here.

Figure 1d shows that the FWHM of the LSPR peak increases with the dopant concentration due to the increasing scattering of the free carriers by the ionized impurities. However, 
this dependence is sub-linear due to the variations of the screening length and the Fermi level with the carrier density. ${ }^{56,57}$ The weak dependence of the FWHM on the NC size suggests that the electrons are scattered by ionized impurities in NCs approximately like in bulk. Remarkably, Fig. 1d shows that the well-known Brooks-Herring theory ${ }^{56,57}$ that describes impurity scattering in a bulk degenerate semiconductor (details in Supporting Information) not only explains the evolution of the FWHM with the dopant concentration but also provides the correct magnitude. Deviations of the calculated FWHMs from the Brooks-Herring law are only visible in the smallest NCs at high doping level (Fig. 1d). The effect of the quantum confinement on the impurity scattering mechanism is therefore weak, which seems counter-intuitive since at the same time the LSPR peak energy is strongly affected. On one hand, this can be understood by the fact that impurity scattering takes place at a length scale corresponding to the mean distance between impurities which is much smaller than the NC diameter. On the other hand, the single-particle oscillators remain quantized due to the confinement, influencing the LSPR frequency. ${ }^{39,40}$

Some experimental studies on $\mathrm{ZnO}$ films have reported that the carrier mobility can sometimes increase at growing dopant concentration, ${ }^{53,58}$ in contradiction with the BrooksHerring theory. This is interpreted by the presence of grain boundaries forming barriers which are reduced at high doping level. This effect cannot be operative in single-crystalline NCs.

The curves of the FWHM vs dopant concentration (Fig. 1d) present small oscillations which can be understood as follows. The LSPR in doped NCs comes from the complex coupling between many single-particle intraband transitions of the free carriers. Even if, for a sufficiently high number of free carriers, the response of the NCs is dominated by collective oscillations at frequencies that only depend on the carrier concentration, ${ }^{37}$ the shape and the width of the LSPR peak are still influenced by the underlying single-particle transitions.

The FWHM of the LSPR peak for single AZO NCs was measured between $75 \mathrm{meV}$ and $197 \mathrm{meV}$, with a median at $150 \mathrm{meV} .{ }^{23}$ The theoretical data are clearly compatible with the 

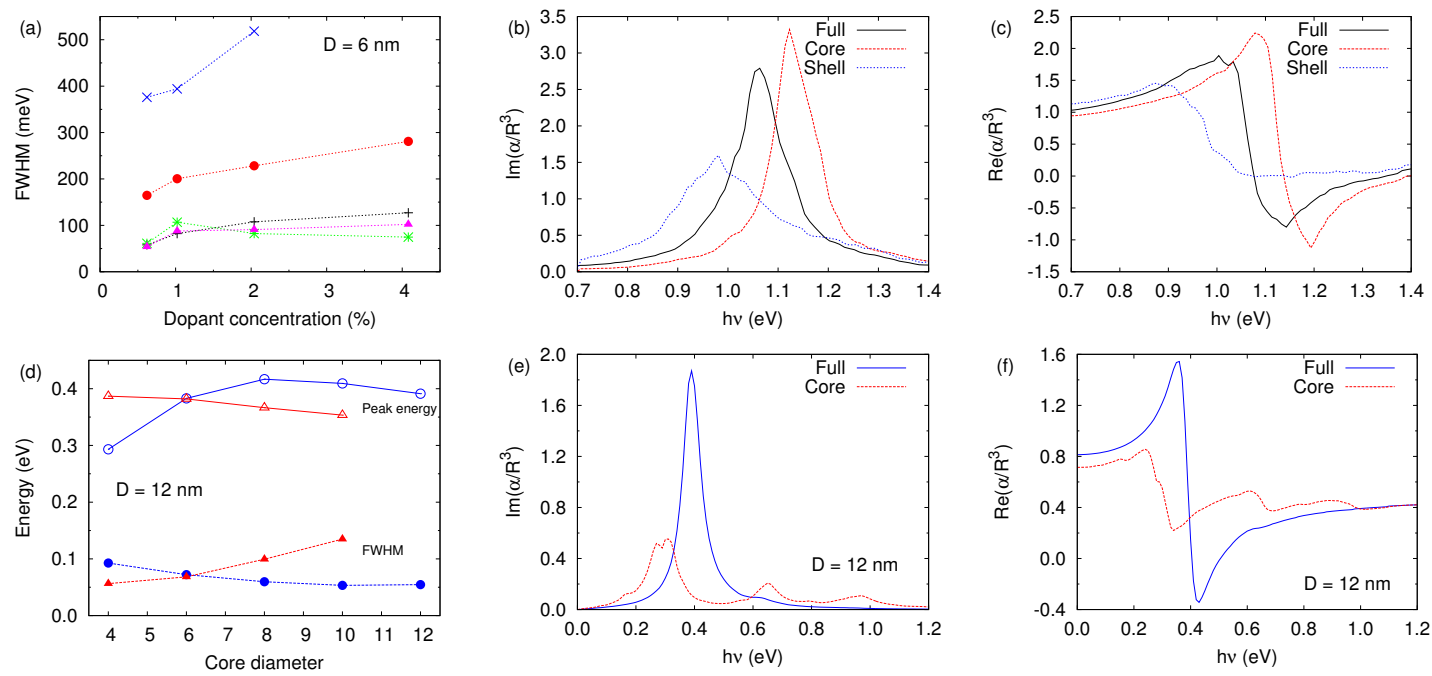

Figure 2: (a) FWHM of the plasmon peak in 6-nm NCs vs active dopant concentration calculated for different situations: uniform doping (black, +), doping in a 4-nm core (green, *), doping in a 1-nm-thick shell (red, circles), uniform doping but with an efficiency of only $30 \%$ (blue, $\times$ ). Magenta triangles: uniform doping but the NCs are embedded in a medium with the same dielectric constant as ZnO. (b) Spectra of $\operatorname{Im}\left(\alpha(h \nu) / R^{3}\right)$ for a 6-nm $\mathrm{NC}$ containing 100 dopants, uniformly doped (Full), doped in a 4-nm core (Core) or doped in a 1-nm-thick shell (Shell). (c) Same as (b) but for the real part $\operatorname{Re}\left(\alpha(h \nu) / R^{3}\right)$. (d-f) Results for 12-nm NCs containing 120 active dopants. (d) Energy (solid lines) and FWHM (dotted lines) of the plasmon peak vs core diameter. The dopants are located either in the core (blue, circles) or in the shell (red, triangles). Imaginary (e) and real (f) parts of $\alpha(h \nu) / R^{3}$ for uniform doping (Full) or doping in a 4-nm core (Core).

lowest experimental values. The present calculations confirm that a FWHM below $100 \mathrm{meV}$ is attainable, which is very promising for applications of doped ZnO NCs in the IR. However, these narrow LSPR peaks are predicted for single uniformly-doped NCs. For example, the FWHM tends to increase in assemblies of NCs due to the coupling between LSPR of neighbor NCs. ${ }^{25}$

It is enlightening to look at physical parameters that influence the FWHM of the LSPR peak. Three features are considered, the dielectric screening by the surrounding medium, the position of the dopants, and the presence of additional scattering centers. It is found that the dielectric constant of the environment has little influence on the FWHM of the LSPR peak whereas it has a strong effect on the LSPR peak energy. Very similar values of the FWHM are obtained for $\varepsilon_{m}$ set at the bulk ZnO value and for $\varepsilon_{m}=1$ (Fig. 2a). This is 
due to the strong screening by the free carriers of the electrostatic potential of the dopants which therefore becomes quite insensitive to the environment. This is also consistent with the weak dependence of the FWHM on the NC size.

The role of the dopant position was studied by considering core-shell ZnO NCs in which the dopants are placed either in the core or in the shell. Examples of spectra for $D=6 \mathrm{~nm}$ are presented in Figs. 2b,c. When the dopants are located in a 5-nm diameter core, the LSPR peak is slightly shifted to higher energy compared to the uniform doping case but the FWHM remains relatively unchanged except at high doping level where the FWHM is slightly reduced (Fig. 2a). On the contrary, when the dopants are placed in a 1-nm-thick shell, the LSPR peak is red-shifted (as found in Ref. ${ }^{39}$ ) and its FWHM is considerably increased. In that case, the electron gas is strongly pushed towards the surface (a typical example is shown in the Supporting Information Fig. S5a) and the scattering by dopant impurities is therefore maximized. The LSPR peak becomes very broad and the real part of $\alpha(h \nu)$ does not reach negative values, i.e., the plasmon is strongly damped. These trends are also found in 12-nm NCs as shown in Fig. 2d which presents the energy and the FWHM of the LSPR peak as functions of the core diameter (shell thickness). A main conclusion of this study is that the doping in a shell near the surface must be avoided if we want to reduce plasmon losses.

An interesting situation occurs when the dopants are placed in a small core at the center of the NC, for example in a 4-nm core of a 12-nm NC (Figs. 2e,f). The imaginary part of $\alpha(h \nu) / R^{3}$ is characterized by three peaks instead of one for uniform doping, and the real part always remains positive. The lowest peak has the highest intensity and is shifted to lower energy compared to the full-doping case (Fig. 2d). The three peaks are attributed to coupled oscillations of different parts of the electron gas. Due to the concentration of the dopant impurities at the center of the $\mathrm{NC}$, an important part of the electron gas is localized in the interior of the NC (Supporting Information Fig. S5a). A deeper analysis of the polarizabilities shows that the lowest-energy peak mostly comes from dipoles close to the 
surface, approximately like in the case of uniform doping (Supporting Information Fig. S5b). On the contrary, the two other peaks at higher energy involve dipoles formed at the interior of the NC.

Figure 2a also shows that the FWHM of the LSPR peak is considerably increased in a situation where only $30 \%$ of the dopants are electrically active, the remaining dopants being still ionized but compensated by positive charges introduced at random positions. These positive charges could simulate the effect of compensating defects (e.g., vacancies, other impurities). In that case, the FWHM of the LSPR peak reaches very high values, above $400 \mathrm{meV}$, due to the high number of fixed charges that induce a relatively unscreened potential.

We have seen that the FWHM of the LSPR peak for single AZO NCs was measured between $75 \mathrm{meV}$ and $197 \mathrm{meV}$, with a median at $150 \mathrm{meV} \cdot{ }^{23}$ In addition, the measured LSPR peak energies can be explained only if the doping efficiency is low, possibly of the order of $20 \%$ (Fig. 1c). However, we conclude from the present calculations that these inactive dopants cannot be all in the ionized state because, otherwise, the FWHM would be too large compared to experiments. In other words, an important fraction of the inactive dopants are probably in a neutral state. For example, dopants could be at surface, antisite or interstitial sites, or associated with another defect to form close pairs. Further experimental and theoretical studies are therefore necessary to investigate situations where dopants behave as neutral centers. In addition, it would be important to determine the relative amount of dopants staying close to the surface since we have seen that they tend to enhance the plasmon damping. If we consider a dopant concentration of $1.5 \%$ and among them $80 \%$ are inactive but neutral, if we assume that neutral defects do not significantly contribute to electron scattering, the FWHM of the LSPR peak must be the one predicted for an active dopant concentration of $0.3 \%$ and therefore, from the present work, should be of the order of $70 \mathrm{meV}$ (Fig. 1d), including a phonon contribution of $10 \mathrm{meV}$. Remarkably, this is close to the lower value found in single-NC experiments of Ref. ${ }^{23}$ 
If we consider the experiments on core/shell-doped NCs, the situation is unclear. In Ref., ${ }^{59}$ a narrowed lineshape is found in Sn-doped $\mathrm{In}_{2} \mathrm{O}_{3}$ NCs when Sn atoms segregated near the surface. In Ref., ${ }^{19}$ the lowest damping is obtained when the core is doped. When the shell is doped, the LSPR linewidth is larger than in core-doped NCs but is smaller than in homogeneously-doped NCs, except for thick shells (>2.5 nm). This complex behavior could be due to a doping efficiency depending on the placement of the dopants ${ }^{7}$ or the thickness of the shell. In addition, the FWHM measured in ensembles of Sn-doped $\mathrm{In}_{2} \mathrm{O}_{3}$ NCs for various doping configurations ranges from 1600 to $3100 \mathrm{~cm}^{-1}$, ${ }^{19}$ while measurements on single homogeneous NCs give values as low as $600 \mathrm{~cm}^{-1} \cdot{ }^{23}$ In these conditions, it is difficult to conclude since the sample heterogeneity may vary depending on the placement of the dopants.

The present work suggests that the LSPR linewidth could be reduced in absence of impurities. However, this narrowing is not seen in the case of photodoped ZnO NCs. ${ }^{40,52}$ Two reasons can be invoked. First, in absence of positive charges inside the NC, the electron gas is strongly pushed at the surface and therefore its oscillation becomes strongly sensitive to surface effects (even more than in the case of shell-doped NCs). Second, the FWHM of the NC ensemble may be determined by inhomogeneous broadening, as found in experiments on single $\mathrm{AZO}$ NCs. ${ }^{23}$

Many experimental studies have reported LSPR peaks characterized by an asymmetric lineshape (for a recent discussion, see Ref. ${ }^{7}$ ). This asymmetry is interpreted by a frequencydependent energy-loss induced by ionized impurity scattering usually described by an analytic expression which can be used to fit the experimental lineshape via free parameters. ${ }^{60}$ However, the physical meaning of these extracted parameters is not clear. For example, in Sn-doped $\mathrm{In}_{2} \mathrm{O}_{3} \mathrm{NCs}$, an important asymmetry is found in Ref. ${ }^{51}$ whereas almost symmetric peaks are reported in Ref. ${ }^{16}$ Furthermore, measurements on single NCs demonstrate that the peak asymmetry is much larger in the ensemble than in single NCs and strongly varies from NC to NC. ${ }^{23}$ For comparison, an analysis of the LSPR peaks calculated in this work 
was performed (Supporting Information, Fig. S6). The calculated peak asymmetry is found to be small, usually below $4 \%$, whereas in the experiments on single AZO NCs, ${ }^{23}$ the peak asymmetry is also small but covers a wider range of values, up to $\pm 10 \%$. On the theoretical side, this discrepancy could come from dynamical correlation effects which are missing in the RPA. On the experimental side, it would be interesting to investigate the effects of the environment and of the sample heterogeneity on the peak asymmetry, effects which are predicted to be large. ${ }^{51}$

In summary, the optical response of $\mathrm{Al}(\mathrm{Ga})$-doped $\mathrm{ZnO}$ NCs was calculated. In the case where the NCs are uniformly doped, their response is characterized by a LSPR at a frequency well given by the classical Drude-Lorentz theory for a $\mathrm{NC}$ diameter of $12 \mathrm{~nm}$ or above. At smaller diameters, the LSPR is shifted to higher energy due to quantum confinement effects. The width of the LSPR peak limited by impurity scattering reaches values below $100 \mathrm{meV}$, in agreement with recent measurements on single ZnO NCs. This width has a weak dependence on the NC size but is considerably increased when dopants are placed in a shell or when additional partially-screened charges are present in the NCs.

\section{Acknowledgement}

This work was supported by the French National Research Agency (ANR) project Gazon (contract ANR-15-CE09-0004-04). The author acknowledges fruitful discussions with Bruno Masenelli and Vincent Sallet.

\section{Supporting Information Available}

Figures showing: (S1) the integral of the optical cross-section vs number of dopants; (S2) the LSPR peak energy vs effective mass; (S3) the evolution of the FWHM vs broadening parameter $\eta$; (S4) absorption spectra calculated in RPA and in single-particle approximation, including in the jellium model; (S5a) electron densities in a $\mathrm{NC}$ uniformly doped, doped in a core or in a shell; (S5b) an analysis of the dipoles in a $\mathrm{NC}$ at main absorption peaks for 
different doping configurations; (S6) the asymmetry ratio versus LSPR peak energy. The Supporting Information also presents a discussion on the effect of effective-mass anisotropy and a derivation of the Brooks-Herring theory in the case of a degenerate semiconductor. This material is available free of charge via the Internet at http://pubs.acs.org/.

\section{References}

(1) Atwater, H. A.; Polman, A. Nat Mater 2010, 9, 205-213.

(2) Anker, J. N.; Hall, W. P.; Lyandres, O.; Shah, N. C.; Zhao, J.; Van Duyne, R. P. Nat Mater 2008, 7, 442-453.

(3) Luther, J. M.; Jain, P. K.; Ewers, T.; Alivisatos, A. P. Nat. Mater. 2011, 10, 361-366.

(4) Scotognella, F.; Della Valle, G.; Srimath Kandada, A. R.; Zavelani-Rossi, M.; Longhi, S.; Lanzani, G.; Tassone, F. Euro. Phys. J. B 2013, 86, 154.

(5) Naik, G. V.; Shalaev, V. M.; Boltasseva, A. Adv. Mater. 2013, 25, 3264-3294.

(6) Liu, X.; Swihart, M. T. Chem. Soc. Rev. 2014, 43, 3908-3920.

(7) Agrawal, A.; Johns, R. W.; Milliron, D. J. Annu. Rev. Mater. Res. 2017, 47, 1-31.

(8) Zhao, Y.; Pan, H.; Lou, Y.; Qiu, X.; Zhu, J.; Burda, C. J. Am. Chem. Soc. 2009, 131, $4253-4261$.

(9) Deka, S.; Genovese, A.; Zhang, Y.; Miszta, K.; Bertoni, G.; Krahne, R.; Giannini, C.; Manna, L. J. Am. Chem. Soc. 2010, 132, 8912-8914.

(10) Scotognella, F.; Della Valle, G.; Srimath Kandada, A. R.; Dorfs, D.; Zavelani-Rossi, M.; Conforti, M.; Miszta, K.; Comin, A.; Korobchevskaya, K.; Lanzani, G.; Manna, L.; Tassone, F. Nano Lett. 2011, 11, 4711-4717. 
(11) Kriegel, I.; Jiang, C.; Rodríguez-Fernández, J.; Schaller, R. D.; Talapin, D. V.; da Como, E.; Feldmann, J. J. Am. Chem. Soc. 2012, 134, 1583-1590.

(12) Rowe, D. J.; Jeong, J. S.; Mkhoyan, K. A.; Kortshagen, U. R. Nano Lett. 2013, 13, $1317-1322$.

(13) Zhou, S.; Pi, X.; Ni, Z.; Ding, Y.; Jiang, Y.; Jin, C.; Delerue, C.; Yang, D.; Nozaki, T. ACS Nano 2015, 9, 378-386.

(14) Ni, Z.; Pi, X.; Zhou, S.; Nozaki, T.; Grandidier, B.; Yang, D. Adv. Opt. Mater. 2016, 4, 646-646.

(15) zum Felde, U.; Haase, M.; Weller, H. J. Phys. Chem. B 2000, 104, 9388-9395.

(16) Kanehara, M.; Koike, H.; Yoshinaga, T.; Teranishi, T. J. Am. Chem. Soc. 2009, 131, $17736-17737$.

(17) Matsui, H.; Furuta, S.; Tabata, H. Appl. Phys. Lett. 2014, 104, 211903.

(18) Schimpf, A. M.; Lounis, S. D.; Runnerstrom, E. L.; Milliron, D. J.; Gamelin, D. R. J. Am. Chem. Soc. 2015, 137, 518-524.

(19) Crockett, B. M.; Jansons, A. W.; Koskela, K. M.; Johnson, D. W.; Hutchison, J. E. ACS Nano 2017, 11, 7719-7728.

(20) Runnerstrom, E. L.; Bergerud, A.; Agrawal, A.; Johns, R. W.; Dahlman, C. J.; Singh, A.; Selbach, S. M.; Milliron, D. J. Nano Lett. 2016, 16, 3390-3398.

(21) Buonsanti, R.; Llordes, A.; Aloni, S.; Helms, B. A.; Milliron, D. J. Nano Lett. 2011, 11, 4706-4710.

(22) Goings, J. J.; Schimpf, A. M.; May, J. W.; Johns, R. W.; Gamelin, D. R.; Li, X. J. Phys. Chem. C 2014, 118, 26584-26590. 
(23) Johns, R. W.; Bechtel, H. A.; Runnerstrom, E. L.; Agrawal, A.; Lounis, S. D.; Milliron, D. J. Nature Communications 2016, 7, 11583 EP -.

(24) Della Gaspera, E.; Bersani, M.; Cittadini, M.; Guglielmi, M.; Pagani, D.; Noriega, R.; Mehra, S.; Salleo, A.; Martucci, A. J. Am. Chem. Soc. 2013, 135, 3439-3448.

(25) Hamza, M. K.; Bluet, J.-M.; Masenelli-Varlot, K.; Canut, B.; Boisron, O.; Melinon, P.; Masenelli, B. Nanoscale 2015, 7, 12030-12037.

(26) Ghosh, S.; Saha, M.; De, S. K. Nanoscale 2014, 6, 7039-7051.

(27) Buonsanti, R.; Milliron, D. J. Chem. Mater. 2013, 25, 1305-1317.

(28) Comin, A.; Manna, L. Chem. Soc. Rev. 2014, 43, 3957-3975.

(29) Faucheaux, J. A.; Stanton, A. L. D.; Jain, P. K. J. Phys. Chem. Lett. 2014, 5, 976-985.

(30) Mattox, T. M.; Ye, X.; Manthiram, K.; Schuck, P. J.; Alivisatos, A. P.; Urban, J. J. Adv. Mater. 2015, 27, 5830-5837.

(31) Agrawal, A.; Singh, A.; Yazdi, S.; Singh, A.; Ong, G. K.; Bustillo, K.; Johns, R. W.; Ringe, E.; Milliron, D. J. Nano Lett. 2017, 17, 2611-2620.

(32) Nat Photon 2012, 6, 714-715.

(33) Klar, T.; Perner, M.; Grosse, S.; von Plessen, G.; Spirkl, W.; Feldmann, J. Phys. Rev. Lett. 1998, 80, 4249-4252.

(34) Jin, Z.; Hamberg, I.; Granqvist, C. G. J. Appl. Phys. 1988, 64, 5117-5131.

(35) Naik, G. V.; Kim, J.; Boltasseva, A. Opt. Mater. Express 2011, 1, 1090-1099.

(36) Monreal, R. C.; Antosiewicz, T. J.; Apell, S. P. J. Phys. Chem. Lett. 2015, 6, 18471853.

(37) Pi, X.; Delerue, C. Phys. Rev. Lett. 2013, 111, 177402. 
(38) Lounis, S. D.; Runnerstrom, E. L.; Llordès, A.; Milliron, D. J. J. Phys. Chem. Lett. 2014, 5, 1564-1574.

(39) Zhang, H.; Kulkarni, V.; Prodan, E.; Nordlander, P.; Govorov, A. O. J. Phys. Chem. C 2014, 118, 16035-16042.

(40) Schimpf, A. M.; Thakkar, N.; Gunthardt, C. E.; Masiello, D. J.; Gamelin, D. R. ACS Nano 2014, 8, 1065-1072.

(41) Delerue, C.; Lannoo, M. Nanostructures: Theory and Modeling; Springer, 2004.

(42) Greenberg, B. L.; Ganguly, S.; Held, J. T.; Kramer, N. J.; Mkhoyan, K. A.; Aydil, E. S.; Kortshagen, U. R. Nano Lett. 2015, 15, 8162-8169.

(43) Meyer, B. K.; Alves, H.; Hofmann, D. M.; Kriegseis, W.; Forster, D.; Bertram, F.; Christen, J.; Hoffmann, A.; Straßburg, M.; Dworzak, M.; Haboeck, U.; Rodina, A. V. Phys. Stat. Sol. (b) 2004, 241, 231-260.

(44) Niquet, Y.-M.; Delerue, C. Phys. Rev. B 2011, 84, 075478.

(45) Pijpers, J. J. H.; Milder, M. T. W.; Delerue, C.; Bonn, M. J. Phys. Chem. C 2010, $114,6318-6324$.

(46) Hedin, L.; Lundqvist, S. In Solid State Physics; Ehrenreich, H., Seitz, F., Turnbull, D., Eds.; Academic: New York, 1969; Vol. 23; p 1.

(47) Lannoo, M.; Delerue, C.; Allan, G. Phys. Rev. Lett. 1995, 74, 3415-3418.

(48) Delerue, C.; Lannoo, M.; Allan, G. Phys. Rev. B 1997, 56, 15306-15313.

(49) Bond, W. L. J. Appl. Phys. 1965, 36, 1674-1677.

(50) Wang, T.; Radovanovic, P. V. J. Phys. Chem. C 2011, 115, 406-413. 
(51) Mendelsberg, R. J.; Garcia, G.; Li, H.; Manna, L.; Milliron, D. J. J. Phys. Chem. C 2012, 116, 12226-12231.

(52) Schimpf, A. M.; Ochsenbein, S. T.; Buonsanti, R.; Milliron, D. J.; Gamelin, D. R. Chem. Commun. 2012, 48, 9352-9354.

(53) Makino, T.; Segawa, Y.; Tsukazaki, A.; Ohtomo, A.; Kawasaki, M. Appl. Phys. Lett. 2005, 8\%, 022101.

(54) Tang, J.; Deng, L. Y.; Tay, C. B.; Zhang, X. H.; Chai, J. W.; Qin, H.; Liu, H. W.; Venkatesan, T.; Chua, S. J. J. Appl. Phys. 2014, 115, 03311.

(55) Germeau, A.; Roest, A. L.; Vanmaekelbergh, D.; Allan, G.; Delerue, C.; Meulenkamp, E. A. Phys. Rev. Lett. 2003, 90, 097401.

(56) Brooks, H. Phys. Rev. 1951, 83, 879.

(57) Chattopadhyay, D.; Queisser, H. J. Rev. Mod. Phys. 1981, 53, 745-768.

(58) Steinhauser, J.; Faÿ, S.; Oliveira, N.; Vallat-Sauvain, E.; Ballif, C. Appl. Phys. Lett. 2007, 90, 142107.

(59) Lounis, S. D.; Runnerstrom, E. L.; Bergerud, A.; Nordlund, D.; Milliron, D. J. J. Am. Chem. Soc. 2014, 136, 7110-7116.

(60) Gerlach, E. J. Phys. C: Solid State Phys. 1986, 19, 4585. 